\title{
Increment in evolution of cellulose crystallinity analysis
}

\author{
Alfred D. French
}

Published online: 11 May 2020

(C) Springer Nature B.V. 2020

Crystallinity analysis is important for practical reasons and related research can offer information on the nature of amorphous cellulose. Two papers in this issue mark a transition in general understanding of cellulose crystallinity analysis. First, a brief review of diffraction crystallinity methods.

Several diffraction methods are used to analyze cellulose crystallinity (Thygesen et al. 2005; Park et al. 2010). The most prevalent, and by far the simplest, is the Segal peak height method (Segal et al. 1959). It had 4871 citations as of this writing, despite frequent use with no attribution or with only citations of secondary publications. Another approach, peak deconvolution, is more effort to carry out and to attribute. Perhaps Hermans and Weidinger (1948) were first to suggest that the area under diffraction peaks be divided by the total area. At present, conventional peak deconvolution involves curve fitting to the observed pattern with the individual visible peaks plus a very broad, but simple, e.g., Gaussian, peak for the amorphous material. Typically, general purpose curve-fitting software is used.

A third method (Rietveld 1969 (16,400 citations); Young 1995) is used for general molecular structure determination of powders as well as occasionally for cellulose crystallinity. The Rietveld method also

\footnotetext{
A. D. French $(\square)$

New Orleans, USA

e-mail: cellulose.editor@gmail.com
}

optimizes variables to fit a diffraction pattern, but it uses all of the diffraction peaks. Unlike peak deconvolution, the Rietveld method includes the smaller peaks that are lost in what appears to be the background or amorphous scattering. These smaller peaks can be visualized by calculating a diffraction pattern for an unrealistically large $(100 \mathrm{~nm})$ model cellulose crystal. Paul Scherrer (1918) showed that peaks are sharp when crystals are large, and broad when crystals are small. When the sharp and separated calculated peaks are broadened to mimic the peaks that arise from model crystals of a size similar to most cellulosic samples (a few nanometers), it appears that much of the intensity formerly attributed to "background" or "amorphous scatter" is just the overlapped intensity from adjacent crystalline peaks (French 2014). The interpretation that the intensity between peaks results from peak overlap, particularly in the region that Segal attributed to only amorphous intensity, casts doubt on the Segal method as a "crystallinity" determination (French and Santiago Cintrón 2013). It also disqualifies a fourth method, "amorphous subtraction" (Thygesen et al. 2005).

The Rietveld method uses the $x-, y$, and $z$ coordinates of the atoms in the crystal structure unit cell such as from Nishiyama et al. (2002) to calculate a diffraction pattern for an ideal crystalline powder. Experimental patterns from cellulose are never ideal, so Rietveld analyses can compensate for the varied unit cell dimensions, inevitable preferred orientation, 
crystallite size, and size-anisotropy, as well as simultaneous presence of two allomorphs or crystalline and amorphous phases (Driemeier and Calligaris 2011; Driemeier 2014). In answering the question "what must be done to modify the ideal pattern so that it matches the experiment?", the values of the refined variables that compensate for the deviations from ideality constitute a thorough description of the crystallinity of a material. However, the number of variables that can be optimized to fit the observed pattern potentially exceeds the number of unique powder data features from most cellulose samples. A disadvantage of the Rietveld method is thus the large number of available variables and small amount of data available in most cellulose powder patterns. In a study with both a laboratory X-ray generator operating in reflection mode and synchrotron radiation in transmission mode (Ling et al. 2019), there was poor agreement on absolute crystallinity values, especially for the crystalline control cotton sample.

One important issue with either peak deconvolution or Rietveld analysis is that a model of the amorphous scattering is needed during curve fitting. Two papers in this issue propose that amorphous cellulose can be best represented by complex equations. These equations are then used along with the crystalline peaks in peak deconvolution studies to construct the total experimental diffraction.

One paper, from Alistair King's group (Rico del Cerro et al. 2020), is focused on the effects of pretreatments on the chemical reactivity of cellulose. An initial objective was to account for any changes in crystallinity, but they independently arrived at an approach similar to the proposal from Jeffrey Catchmark's group (Yao et al., 2020). In the case of Yao et al. the equation representing the amorphous component was structured as an eighth order Fourier series, whereas Rico del Cerro et al. used nine Gaussian functions to represent the amorphous fraction (Fig. 1).

The shared essence of both papers is that the amorphous contribution to the diffraction pattern is a more complex shape than the simple single broad Gaussian used by so many authors. This permits fitting to data from amorphous samples such as a standard prepared from pulp regenerated from trifluoroacetic acid (Rico del Cerro et al.) or ball milled examples of several materials (Yao et al.). Both groups used commercial peak fitting software for their analyses.
Such complex representations should be an advance in the analysis of cellulose diffraction patterns, at least compared to the use of a simple single peak to represent amorphous scattering.

Rather than being truly novel, the methods used in these two papers signal the attainment of a critical momentum towards better treatment of the amorphous component in crystallinity analysis. Previously, Thygesen et al. (2005) used a 10-parameter Chebychev polynomial function that included background; that pattern peaks at about $16^{\circ} 2 \theta$. Ju et al. (2015) reported an amorphous curve from phosphoric acidtreated nanocrystalline cellulose. It has three maxima, one at essentially the same location of $20.5^{\circ}$ of most other proposals, with additional peaks reported at $38.87^{\circ}$ and $80.90^{\circ}$ (all $2 \theta$ values are from $\mathrm{Cu} \mathrm{K \alpha}$ radiation). A truncated version of that pattern is also in Fig. 1. They modeled the amorphous component with three different Gaussians. Ju et al.'s peak at $20.6^{\circ}$ is considerably sharper than that of Yao et al. but follows the Rico del Cerro et al. peak near the maximum, see Fig. 1.

Another approach (Le Bail 1995) has been to use a calculated pattern from the same material but with a very small crystal size. Duchemin (2017) used a small crystal from Gardiner and Sarko's (1984) cellulose IV $_{\text {I }}$ and Ling et al. (2019) used Langan et al.'s (2001) cellulose II with a very large peak width at half maximum (or Full Width and Half Maximum, pwhm or FWHM), e.g. $9^{\circ}$. The Scherrer equation translates a $9^{\circ}$ pwhm to a crystallite size of $\approx 1.2 \mathrm{~nm}$. In the quest to better understand the structure of "amorphous" cellulose, the calculated patterns from $\alpha$-glucose (Fronczek 2016) and $\beta$-cellobiose (Chu and Jeffrey 1968) are shown along with the Yao et al. pattern and the wide-peak pattern for cellulose II in Fig. 1. None of these proposed patterns are identical, but there is enough similarity to clarify that a simple Gaussian peak does not adequately represent amorphous cellulose. Some wide-peak patterns from crystal structures of related small molecules were fairly different from the patterns in Fig. 1.

To the extent that widely available software is used, the type of amorphous representation used will depend on the available functions. In FitYK, for example, mathematical functions are available and some provisions are present for crystallographic analyses such as a Pawley fit that adjusts all expected peak intensities for a given unit cell to best fit a diffraction pattern. In 


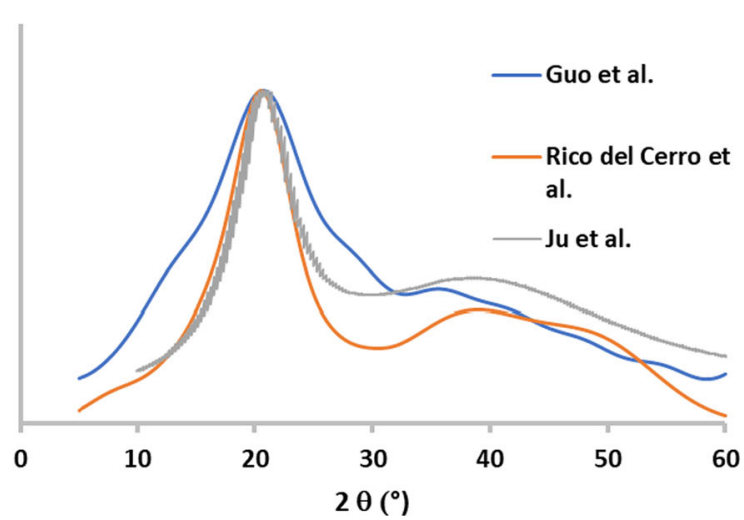

Fig. 1 Left: Derived intensity for amorphous cellulose (Yao et al. 2020; Rico del Cerro et al. 2020) and the fitted data of Ju et al. (2015). Right: Comparison of calculated intensities for the amorphous diffraction pattern (Yao et al. 2020) and from

the MAUD Rietveld program (Lutterotti 2010), background representations that could also be applied to amorphous scattering include polynomials, distribution functions, and point-interpolated splines. However, the MAUD author concurs with Le Bail (1995) and recommends describing the amorphous pattern using an extra phase composed of the primary scatterer, i.e., crystallites of about one to three unit cells in size (Lutterotti, 2012).

Other free and commercial Rietveld software offers different functions. Some creativity could be utilized to import the basic results of the fitted equations in Rietveld software that lacked functionality to use a Fourier series. For example, the Yao et al. equation could be solved for a range of $2 \theta$ and those data could be input into the Rietveld program as a data file and a wide-peak structure could be fit to it. A simple fit of the wide-peak cellulose II pattern to the Yao et al. pattern, varying only the scale factor and crystallite size, resulted in a crystallite size of $0.95 \mathrm{~nm}$ and a discrepancy (R) of $5.24 \%$. The resulting crystallite size could then be used with the cellulose II crystal information file to represent the Yao et al. amorphous fraction.

What should the editorial policy of Cellulose be, given the situation where the three most widely used methods have limitations? After all, the indicator of amorphous component for the Segal method is substantially influenced by overlap of crystalline peaks. The peak deconvolution method uses only visible peaks, and is likely to attribute some of the crystalline intensity to amorphous component or background. The Rietveld method has a large number

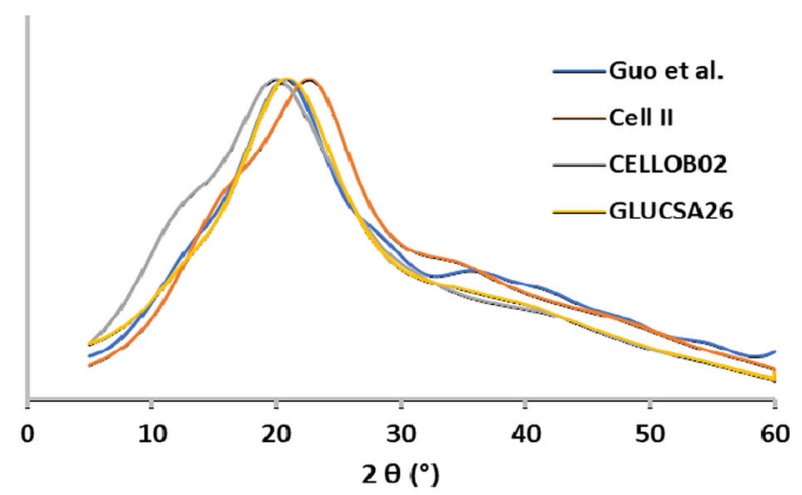

pwhm $=9^{\circ}$ calculations for cellulose II (Langan et al. 2001), cellobiose (Chu and Jeffrey 1968) and $\alpha$-glucose (Fronczek 2016)

of variable parameters and an unclear number of unique data points to guide the refinement.

The answer is that we ask all authors of studies that include crystallographic work to be aware of the above situations and meet some standards. Nomenclature (diffraction peak labels) for the cellulose allomorphs must conform to the conventions used in French (2014). The minimum intensity for the highest peak should be at least 500 counts to avoid excess noise. Data from a blank run including the sample holder should be subtracted from the data for experimental samples. (A further adjustment may be helpful.) The method must be carried out correctly. There is a horrible error in the literature for the Segal method, where the overlapped (1-10)/(110) peak is described as the "amorphous peak" to go with the (200) "crystalline peak". The minimum between these peaks is correct, not a peak. Please be alert for this error and stop its spread in the literature. Note also that the application of the Segal method to cellulose II (Azubuike et al. 2012) designates a minimum at about $16^{\circ} 2 \theta$ to represent the amorphous content. According to Fig. 1 the amorphous scattering is not near its maximum intensity at $16^{\circ}$, so intensity at this point also includes the peak overlap and intensity from residual cellulose I. We encourage authors who are able to move beyond the Segal method, and to document the deconvolution, preferably graphically, from the peak deconvolution method. Similarly, the refinement variables in the Rietveld method should be recorded so that the work could be reproduced. Data are welcome as Supplementary Information. 
There is room for more investigation of amorphous diffraction. As the methods improve, can we establish whether all complete preparations of amorphous cellulose give the same diffraction pattern? Yao et al. (2020) show experimental diffraction patterns of cellooligomers that are essentially identical with the ball-milled samples of several kinds of cellulose, but can the apparent gap between the ball-milled samples and the dissolved and regenerated cellulose ( $\mathrm{Ju}$ et al., Rico del Cerro) be closed?

Acknowledgments Helpful comments on drafts of the editorial were kindly provided by Jeffrey Catchmark, Alistair King, Michael Santiago Cintrón, Benoît Duchemin, and Wenqing Yao.

\section{References}

Azubuike CP, Rodríguez H, Okhamafe AO, Rogers RD (2012) Physicochemical properties of maize cob cellulose powders reconstituted from ionic liquid solution. Cellulose 19:425-433. https://doi.org/10.1007/s10570-011-9631-y

Chu SSC, Jeffrey GA (1968) The refinement of the crystal structures of $\beta$-D-glucose and cellobiose. Acta Crystallogr B 24:830-838. https://doi.org/10.1107/S0567740868003250

Driemeier C, Calligaris GA (2011) Theoretical and experimental developments for accurate determination of crystallinity of cellulose I materials. J Appl Crystallogr 44:184-192. https://doi.org/10.1107/S0021889810043955

Driemeier C (2014) Two-dimensional Rietveld analysis of celluloses from higher plants. Cellulose 21:1065-1073. https://doi.org/10.1007/s10570-013-9995-2

Duchemin B (2017) Size, shape, orientation and crystallinity of cellulose $\mathrm{I} \beta$ by $\mathrm{X}$-ray powder diffraction using a free spreadsheet program. Cellulose 24:2727-2741. https://doi. org/10.1007/s10570-017-1318-6

French AD, Santiago Cintrón M (2013) Cellulose polymorphy, crystallite size, and the Segal crystallinity index. Cellulose 20:583-588. https://doi.org/10.1007/s10570-012-9833-y

French AD (2014) Idealized powder diffraction patterns for cellulose polymorphs. Cellulose 21:885-896. https://doi. org/10.1007/s10570-013-0030-4

Fronczek FR (2016) GLUCSA26, $\alpha$-D-glucose, CSD Communication, Cambridge Crystallographic Data Centre (not otherwise published)

Gardiner ES, Sarko A (1984) Packing analysis of carbohydrates and polysaccharides. 16 The crystal structures of celluloses $\mathrm{IV}_{\mathrm{I}}$ and $\mathrm{IV}_{\mathrm{II}}$. Can J Chem 63:173-180. https://doi.org/10. 1139/v85-027

Hermans PH, Weidinger A (1948) Quantitative X-ray investigations on the crystallinity of cellulose fibers: a background analysis. J Appl Phys 19:491-506. https://doi.org/ 10.1063/1.1698162

Ju X, Bowden M, Brown EE, Zhang X (2015) An improved X-ray diffraction method for cellulose crystallinity measurement. Carbohydr Polym 123:476-481. https://doi.org/ 10.1016/j.carbpol.2014.12.071
Langan P, Nishiyama Y, Chanzy H (2001) X-ray structure of mercerized cellulose II at $1 \AA$ resolution. Biomacromol 2:410-416. https://doi.org/10.1021/bm005612q

Le Bail A (1995) Modelling the silica glass structure by the Rietveld method. J Non-Cryst Solids 183:39-42. https:// doi.org/10.1016/0022-3093(94)00664-4

Ling Z, Wang T, Makarem M, Santiago Cintrón M, Cheng HN, Kang X, Bacher M, Potthast A, Rosenau T, King H, Delhom CD, Nam S, Edwards JV, Kim SH, Xu F, French AD (2019) Effects of ball milling on the structure of cotton cellulose. Cellulose 21:885-896. https://doi.org/10.1007/ s10570-018-02230-x

Lutterotti L (2010) Total pattern fitting for the combined sizestrain-stress-texture determination in thin film diffraction. Nucl Instrum Meth B 268:334-340. https://doi.org/10. 1016/j.nimb.2009.09.053

Lutterotti L (2012) Quantitative phase analysis: method developments. In: Kolb U, Shankland K, Meshi L, Avilov A, David WI (eds) Uniting electron crystallography and powder diffraction. Springer, pp 233-242. https://doi.org/ 10.1007/978-94-007-5580-2_21

Nishiyama Y, Langan P, Chanzy H (2002) Crystal structure and hydrogen-bonding system in cellulose $\mathrm{I} \beta$ from synchrotron $\mathrm{X}$-ray and neutron fiber diffraction. J Am Chem Soc 124(31):9074-9082. https://doi.org/10.1021/ja0257319

Park S, Baker JO, Himmel ME, Parrilla PA, Johnson DK (2010) Cellulose crystallinity index: measurement techniques and their impact on interpreting cellulase performance. Biotechnol Biofuels 3:10. https://doi.org/10.1186/1754-6834-3-10

Rico del Cerro DR, Koso TV, Kakko T, King AWT, Kilpeläinen I (2020) Crystallinity reduction and enhancement in the chemical reactivity of cellulose by non-dissolving pretreatment with tetrabutylphosphonium acetate. Cellulose. https://doi.org/10.1007/s10570-020-03044-6

Rietveld HM (1969) A profile refinement method for nuclear and magnetic structures. J Appl Crystallogr 2:65-71. https://doi.org/10.1107/S0021889869006558

Scherrer P (1918) Bestimmung der Grösse und der inneren Struktur von Kolloidteilchen mittels Röntgenstrahlen (Determination of the size and internal structure of colloid particles using X-rays). Göttinger Nachrichten Gesell 2:98-100

Segal L, Creely JJ, Martin AE Jr, Conrad CM (1959) An empirical method for estimating the degree of crystallinity of native cellulose using the X-ray diffractometer. Text Res J 29:786-794. https://doi.org/10.1177/ 004051755902901003

Thygesen A, Oddershede J, Lilholt H, Thomsen AB, Ståhl K (2005) On the determination of crystallinity and cellulose content in plant fibres. Cellulose 12:563-576

Yao W, Weng Y, Catchmark J (2020) Improved cellulose $\mathrm{X}$-Ray diffraction analysis using Fourier series modeling. Cellulose. https://doi.org/10.1007/s10570-020-03177-8

Young RA (1995) The Rietveld Method. International Union of Crystallography Monographs on Crystallography. Oxford pp 312. ISBN: 9780198559122

Publisher's Note Springer Nature remains neutral with regard to jurisdictional claims in published maps and institutional affiliations. 